\title{
Pensamiento reflexivo e investigador en educación. Aspectos a tener en cuenta en la formación del profesorado
}

\author{
Reflective and Inquary Thinking in Education. Aspects to consider in teacher education
}

\author{
Fernández-Fernández, Samuel ${ }^{(1)}$; Arias-Blanco, José-Miguel ${ }^{(1)}$; Fernández-Alonso, \\ Rubén $^{(1)}$ (2); Burguera-Condon, Joaquín ${ }^{(1)}$ \& Fernández-Raigoso, Marcelino ${ }^{(1)}$
}

(1) Universidad de Oviedo (España)

(2) Consejería de Educación y Cultura del Principado de Asturias (España)

\begin{abstract}
Resumen
Formar al profesorado como profesionales reflexivos e investigadores debe ser una prioridad para un sistema educativo que busca la mejora de su alumnado en el manejo de información compleja y la resolución de problemas de forma creativa y divergente. El educador reflexivo, tal como es percibido por el profesorado español que participa en el estudio TALIS (OECD, 2014), ha sido distribuido en una escala o índice TRI que permite mostrar la frecuencia de participación en actividades formativas que facilitan un "Desarrollo profesional reflexivo" (DPR). La mitad de la muestra considera que su desarrollo profesional incluye actividades formativas ocasionales de carácter reflexivo. Se identifican, mediante análisis multinivel, los factores individuales y de centro asociados al perfil docente de educador reflexivo, que se corresponden con un atributo individual vinculado a una formación en red o de colaboración docente en torno a un centro con liderazgo pedagógico y control evaluativo. Esto supone para el docente una mayor dedicación e intensidad, una autopercepción de efectividad profesional y dominio sobre los procesos de enseñanza-aprendizaje que utiliza con su alumnado. El índice DPR se muestra consistente en la representación de un perfil docente que favorece la efectividad de los procesos de aula. Por la situación que presenta España en la estimación del índice DPR, consideramos de interés hacer evolucionar los modelos de formación inicial y continua del profesorado hacia un enfoque que potencie las capacidades reflexiva, investigadora y colaborativa de nuestro profesorado y estudiantes.
\end{abstract}

Fecha de recepción 13 de Junio 2016

Fecha de aprobación 22 Noviembre 2016

\section{Fecha de} publicación 22 Noviembre 2016

Palabras clave: Educador reflexivo, profesorado investigador, formación de profesorado.

\begin{abstract}
Training teachers as reflective practitioners and researchers should be a priority for an educational system that seeks to improve their students in managing complex information and solving problems creatively and divergently The reflective educator, as perceived by the Spanish teachers participating in TALIS, has been distributed on a scale or TRI index that allows us to show the frequency of participation in educational activities that facilitate a reflective professional development (DPR). Thus we see that half of the sample believes that his professional development includes occasionally training activities of reflective character. Identified by multilevel analysis, the personal and school factors associated with the reflective teacher educator profile, correspond to an individual attribute but linked to a collaborative network for training teacher around a school center with instructional leadership and evaluative control. This means for teachers more dedication and intensity, but also results in a self-perception of professional effectiveness and control over the processes of teaching and learning using with their students. DPR index is shown as consisting in the representation of a teaching profile that favors the effectiveness of classroom processes. According to the situation of Spain in estimating the DPR index, we consider of interest to evolve the current model of initial and continuing teacher education towards an approach that enhances the reflective and collaborative research capabilities of our faculty and students.
\end{abstract}

\section{Reception \\ Date}

2016 June 13

Approval

Date

2016

November 22

Publication

Date:

2016

November 22

Keywords: Reflective Teaching, Teacher Researchers, Teacher Education Programs.

Las conclusiones de las evaluaciones diagnósticas internacionales del rendimiento educativo de los estudiantes vienen incidiendo en la necesidad de introducir cambios en aquellas variables que más influyen en el aprendizaje e indican que la formación 
profesional del profesorado es uno de 1 los principales aspectos que deben ser revisados.

La mejora de resultados educativos requiere cambios en la enseñanza por lo que conviene iniciar esta aportación repasando las evidencias de la investigación sobre los elementos que tienen mayor influencia en el rendimiento. Hattie (2003) indica que las principales fuentes de varianza se pueden concretar en seis. En primer lugar lo que el estudiante aporta personalmente al proceso de aprendizaje, es decir, su capacidad, con un 50\%. En segundo lugar el profesorado con el 30\%. El tercero de los factores es la influencia del hogar, medida por el nivel de expectativas y apoyo familiar, que contribuye con un 5-10\% de la varianza. Las características escolares, como el tamaño de clase y los recursos disponibles, las influencias de los compañeros, operativizadas en plan positivo por la co-enseñanza y en el negativo por los problemas de convivencia tienen una aportación similar a esta última, un 5-10\% respectivamente.

Por lo tanto, el profesorado, con un 30\% de influencia, constituye el factor que la investigación plantea que se debe optimizar. Como indica Hattie, aunque casi todo lo que se hace en nombre de la educación tiene un efecto positivo sobre el rendimiento, los mayores logros son los relativos al profesorado: la retroinformación (tamaño de efecto de 1,13 desviaciones estándar), la enseñanza dirigida $(0,82)$, el apoyo correctivo $(0,65)$, el clima de aula $(0,56)$, la tutoría de pares $(0,50)$, la calidad de las tareas de casa $(0,43)$ y el estilo de enseñanza $(0,42)$, en particular la indagación científica (Anderson, 2002).

Con referencia al estilo y a la metodología de enseñanza, se considera una característica del profesorado experto conseguir un claro equilibrio entre la enseñanza centrada en el estudiante $y$ el contenido a aprender, preocuparse porque sus enseñanzas sean consideradas útiles y buscar nuevas formas de trabajar en el aula de manera que sus estudiantes empleen más tiempo en hacer tareas que en escuchar al profesor.
John Dewey, en los pasados años 30, exponía su enfoque del profesorado como profesionales reflexivos así como la necesidad de construir la teoría desde la práctica. De acuerdo con Dewey, la educación es más adecuada cuando se practica como un proceso de indagación y cuando el profesorado utiliza el enfoque científico en su análisis. Igualmente mantiene que su doble papel en el aula es el de profesional de la enseñanza e investigador de problemas reales que debe resolver mediante la superación de los obstáculos que puedan existir para su comprensión y manejo.

Stenhouse, veinte años después, mantenía que el profesorado debía ser competente para evaluar su teoría sobre la enseñanza con el objetivo de cambiar su propia práctica. Desde entonces, han sido muchos los autores que abogan por la necesidad de reflexión en la acción para todos aquellos profesionales (arquitectos, abogados, profesores,...) que trabajan con problemas de la vida real de naturaleza compleja.

Cochran-Smith y Lyttle (2009) describen dos tipos de profesorado investigador, el conceptual y el empírico. El primero es teórico y trabaja con análisis interpretativos; el segundo explora y analiza datos, pero ambos se muestran reflexivos, analíticos y críticos en sus enseñanzas y también, más abiertos a su desarrollo profesional.

Esta caracterización apunta hacia la importancia del perfil profesional que denominamos de profesorado investigador, indagador o reflexivo (Schön, 1987; CochranSmith \& Lytle, 2009). En este mismo sentido, Stremmel (2007) pone de manifiesto el valor del profesor investigador puesto que la enseñanza debe considerarse un proceso que requiere una permanente actitud de análisis y cambio. Actuar como profesor investigador implica pensar y reconstruir el significado de ser educador, la forma de trabajar con el alumnado, comprender el funcionamiento de las enseñanzas y desarrollar un aprendizaje más experiencial. Así, un profesor o profesora debe ser, ante todo, investigador y utilizar de forma 
combinada en su enseñanza, la reflexión y la acción.

\section{Reflexión e investigación en la formación del profesorado}

El concepto de "pensamiento reflexivo", atribuido a Dewey, se describe como un procesamiento de ideas que la Educación se debería esforzar en cultivar, y se ha desarrollado en forma de taxonomía de cuatro escalas (Kembera et al., 2008): acción habitual o no reflexiva, comprensión, reflexión $\mathrm{y}$ reflexión crítica. La primera ocurre en aquella práctica profesional que sigue un procedimiento de rutina, sin mediar ningún análisis. La segunda categoría es la típica de las clases de teoría e implica el intento de comprensión de un concepto, por ejemplo, el significado subyacente en una expresión oída o leída, lo que supone baja retención o asimilación en el conocimiento de la persona, ya que no se analiza la aplicación personal y práctica de lo comprendido. El proceso de reflexión supone trabajar con un concepto en relación a experiencias personales que requiere aplicación de la teoría $\mathrm{y}$, por lo tanto, adecuación e inferencias que trascienden la mera comprensión. Finalmente, la reflexión crítica conlleva una perspectiva transformadora que estos autores consideran más propia del estudiante en formación que de los profesionales con creencias y rutinas asentadas.

Dana y Yendol-Hoppey (2009) en su Guía del Educador Reflexivo en la Investigación de Aula, presentan técnicas para facilitar la práctica indagatoria al profesional de la educación, tanto para el trabajo colaborativo, como para recoger información y desarrollar una secuencia de pasos desde la descripción, la toma de sentido, la interpretación y la participación en el análisis de la información. El profesorado investigador utiliza, en primer lugar, metodologías cualitativas que le permiten examinar la práctica docente desde dentro. La observación directa, la recogida de notas o registro de incidentes, la escritura de diarios o la realización de entrevistas se consideran adecuados para comprender la compleja naturaleza de la enseñanza y el aprendizaje. De acuerdo nuevamente con Dewey (1985), la educación obtiene mejores resultados cuando el profesorado emplea el método científico para cuestionar y mejorar su práctica de tal modo que, en su doble condición de profesional e investigador en el aula, se enfrenta a problemas reales que le permiten comprender y sopesar a diario por qué las cosas son como son.

La evidencia sugiere que el profesorado que se ha formado y practicado en investigación educativa mantiene un enfoque más reflexivo en su trabajo de aula, más abierto y dispuesto a un desarrollo profesional más activo (Rust, 2007). Como indica Stremmel (2007), el valor real de implicarse en la investigación de aula, en cualquier nivel educativo, es que permite repensar y reconstruir la forma de relación con el alumnado. Esta actitud de investigación en el profesorado tiene el potencial de mostrar que aprender a enseñar es inherente al aprendizaje por descubrimiento y que el gran objetivo de la investigación de aula es capacitar al profesorado para la comprensión de su actividad profesional mediante la indagación reflexiva $^{[1]}$. En cualquier caso, el proceso de reflexión e investigación del profesorado debe ser riguroso y asumir los criterios de valor de la investigación: credibilidad ante aquellos que son competentes para juzgar el trabajo; transferibilidad, que permita el intercambio de la experiencia de unos educadores a otros; fiabilidad de los procedimientos y consistencia de resultados y logros mediante confirmación objetiva.

La discusión teórica en educación sobre la práctica reflexiva es amplia. Schön (1987) ha acotado con precisión el concepto al considerarla como la actividad socioprofesional por la que el profesorado adapta el conocimiento a situaciones específicas. La investigación sobre enfoques de desarrollo profesional de tipo reflexivo y colaborativo indica que al emplear esas estrategias, el profesorado es capaz de mejorar su práctica docente en mayor medida que cuando intenta hacerlo de forma aislada o mediante una 
formación permanente de tipo tradicional (Darling-Hammond \& Bransford, 2005).

Sparks-Langer y Berstein (1991) argumentan sobre tres elementos considerados importantes para la formación del pensamiento reflexivo en el profesorado. El primero es el elemento cognitivo, que describe cómo el profesor procesa información y toma decisiones. El segundo es el elemento crítico, centrado sobre lo experiencial, las creencias y valores, y las implicaciones sociales, como sustancias que conducen el pensamiento. El tercer elemento de la reflexión lo forman las narraciones de los propios profesores que reflejan las interpretaciones de los sucesos que acontecen en su particular contexto.

En cuanto a la reflexión cognitiva, plantean que es posible enseñar al profesorado novel los esquemas de los expertos pero se subvertiría lo aprendido del constructivismo de que cada uno debe construir su propio significado y, también, el planteamiento de la cognición contextual, ya que el profesorado experto situaría sus esquemas en su propia experiencia.

Promover la reflexión narrativa se fundamenta en la importancia de agrupar expresiones y conceptos mediante la capacidad del ser humano de contar historias. Los profesores en formación construyen relatos ganando en reflexión sobre sus experiencias ya que muestran lo que motiva a la acción a la vez que proporcionan una detallada casuística sobre la enseñanza y en especial, claridad de ideas debido al autoanálisis de su actividad.

No obstante, formar al profesorado para trabajar mediante una enseñanza reflexiva es algo más que una cuestión técnica ya que se deben adquirir nuevas competencias y asumir el rol de gestor de aprendizajes en el que la dimensión cultural del contexto escolar juega un papel importante. Dado que la formación de profesorado reflexivo se considera uno de los objetivos fundamentales a conseguir en la nueva formación docente (NCATE, 2008), las demandas de cambio en los modelos de formación se dirigen a promover la indagación en la enseñanza y la reflexión sobre la práctica, apareciendo propuestas, como la de Freese
(2006) de que la formación inicial del profesorado se base en introducir a un descubrimiento que permita a los estudiantes analizar la enseñanza a través de la reflexión .

Esta práctica deliberativa y reflexiva se caracteriza por identificar problemas y generar y probar soluciones. Se considera también la reflexión como la interacción de experiencias con el análisis de creencias sobre esas experiencias (Newell, 1996). Tal como indican Etscheid, Curran y Sawyer (2012), al modelo básico de Schön (1987) se han ido añadiendo planteamientos diversos como el de Jay y Johnson (2002) y Ward y McCotter (2004). Los primeros consideran una modalidad de reflexión descriptiva para la valoración personal de la acción en el aula; una comparativa, que plantea puntos de vista alternativos; y otra crítica, que cuestiona dimensiones morales y políticas de la educación. Los segundos distinguen reflexión básica, que analiza el impacto de la práctica o la experiencia sobre la formación del profesor, pero con poca reflexión personal; técnica, que valora y promueve la reflexión sobre temas concretos de la enseñanza; dialógica, que pone en funcionamiento perspectivas divergentes; $y$, finalmente, reflexión transformadora, que pone en funcionamiento elementos culturales, históricos, éticos y morales para concienciar de cambios en la práctica

\section{Método}

\section{Objetivos}

Por todo lo expuesto, se considera que promover cambios efectivos en el aula y el centro educativo requiere construir y evaluar la teoría mediante una indagación intencional y sistemática, con el objetivo de mejorar la comprensión de los procesos de enseñanzaaprendizaje ya que los efectos educativos apreciados al seguir una enseñanza reflexiva o indagatoria son claros en la mejora de la cognición (Scruggs \& Mastropieri, 1994).

Este cambio de rol en la metodología docente lleva implícito conocer, en primer lugar, la percepción del profesorado sobre su desarrollo profesional, para lo cual se organiza 
un modelo analítico basado en el estudio TALIS, a nivel individual y de centro, sobre variables de entrada, proceso y producto, de acuerdo con el diseño metodológico que se expone más adelante.

El presente estudio tiene cuatro objetivos principales:

- Analizar la situación de España en el índice internacional "Desarrollo profesional efectivo” (TEFFPROS) estimado en la evaluación TALIS.

- Construir un índice paralelo sobre la muestra española que permita describir el constructo "Desarrollo profesional reflexivo" (DPR).

- Estimar la incidencia que el "Desarrollo profesional reflexivo" tiene entre el profesorado de enseñanza secundaria en España.

- Analizar qué factores personales y profesionales se asocian al profesorado que puntúa alto en el índice "Desarrollo profesional reflexivo".

\section{Población y Muestra}

Se utilizan en este análisis las respuestas de la muestra de España del profesorado participante en el estudio TALIS. Sus características principales son las siguientes: profesorado mayoritariamente femenino, un tercio tiene más de 50 años, amplia experiencia docente (el $40 \%$ acumula más de 20 años de docencia) y relativa continuidad (un tercio lleva más de 11 años en el mismo centro).

Las variables relativas a centros corresponden a las respuestas de los directores que participaron en el estudio. Un primer análisis revela que estas funciones organizativas son desarrolladas por profesorado con mucha experiencia (más de dos tercios tiene más de dos décadas de ejercicio profesional). Destaca la estabilidad en estas funciones directivas (una cuarta parte lleva más de 11 años como director) y la prevalencia de varones desempeñando estas tareas, cuando la mayoría del profesorado son mujeres.

\section{Instrumento}

Definición de la variable dependiente "Desarrollo profesional reflexivo" y de las variables independientes seleccionadas para su predicción.

\section{Variable dependiente: "Desarrollo profesional reflexivo"}

En correspondencia con los perfiles de profesorado buscados, se define el "Desarrollo profesional reflexivo" en función de la información que proporciona la encuesta TALIS relativa a la participación en actividades de formación que presentan las siguientes características:

Formación dirigida a la mejora de la organización escolar y al desarrollo de métodos o recursos de un área o materia. Por tanto, no surge de un interés particular del profesorado, sino que, se trata de actividades que se realizan conjuntamente con otros compañeros del centro o de la materia.

Es una formación que exige que el profesorado participe activamente y que construya su propio conocimiento, por lo que es necesario que dicha formación sea inseparable de estudios e investigaciones sobre el tema de interés.

Es una actividad planificada, que necesita para su desarrollo de un tiempo de estudio y que no se limita a una acción puntual.

Para valorar en qué medida las actividades de formación permanente de los docentes reúnen las mencionadas características, el cuestionario TALIS formulaba cuatro cuestiones relacionadas y un primer análisis de los datos permite afirmar que el profesado español se preocupa por realizar acciones de formación continua, puesto que el $80 \%$ indica que ha participado en alguna actividad en los doce meses anteriores a la encuesta, en tanto que un $15 \%$ reconoce no haber realizado ninguna acción de este tipo.

A pesar de la cotidianeidad de la formación permanente, no todas las actividades de formación presentan las características propias del DPR. Sólo el $21 \%$ indica que sus actividades de formación incluyen la realización conjunta de estudios e 
investigaciones, y sólo uno de cada cuatro encuestados señala que han sido acciones sostenidas en el tiempo. Esto refuerza la idea de que, si bien el profesorado español invierte tiempo y esfuerzo en su formación permanente, no toda la formación ofertada o seleccionada reúne las condiciones para facilitar el "Desarrollo profesional reflexivo".

\section{Variables independientes}

De acuerdo con lo señalado, los predictores se clasifican en dos niveles, individual (profesorado) e institucional (centro).

Entre las variables individuales distinguimos factores de entrada y/o contexto y variables de proceso. Las primeras son sexo, experiencia docente, asignatura o materia impartida y nivel de estudios del profesorado.

Incluimos, a su vez, catorce variables de proceso que agrupamos en cinco categorías: percepciones profesionales, gestión de los procesos instructivos del aula, formación continua, participación y cooperación docente y tiempo de dedicación.

$\mathrm{Al}$ igual que ocurría con las variables a nivel de profesorado, los factores institucionales de centro distinguen entre variables de entrada y proceso. Las variables de entrada son la titularidad, el tamaño, el nivel socioeconómico y cultural del centro y la ratio alumnado/profesorado. Las de proceso, por su parte, son el promedio de horas trabajadas por centro, el uso de la evaluación para dar retroalimentación, la autonomía curricular, la autonomía escolar en la gestión: (contratación y salarios) y el liderazgo pedagógico.

\section{Análisis de datos}

Para lograr el primer objetivo se realiza una comparación de medias del índice "Desarrollo profesional efectivo" (TEFFPROS), considerado en la encuesta de TALIS. El índice TEFFPROS está construido con los mismos ítems que conforman el constructo "Desarrollo profesional reflexivo" del presente estudio.

El segundo objetivo del trabajo consiste en validar una escala paralela al TEFFPROS, pero centrada en la muestra de España. Este índice busca expresar la probabilidad del profesorado español de participar en actividades de formación que faciliten un "Desarrollo profesional reflexivo" (DPR).

Una vez construida la escala "Desarrollo profesional reflexivo" mediante el ajuste TRI, se aborda el tercer objetivo del estudio: estimar la incidencia que este constructo tiene entre el profesorado de enseñanza secundaria en España. Para ello es preciso determinar los puntos de corte que permitan situar a cada docente en un grupo en función de su puntuación en dicha escala. Una vez establecidos los puntos de corte se crean tres categorías según el nivel de compatibilidad con la definición de "Desarrollo profesional reflexivo”. Las categorías o grupos docentes se definen del siguiente modo:

Sin evidencias de formación compatible con el "Desarrollo profesional reflexivo". Profesorado que participa en actividades formativas cortas y puntuales, sin conexión con los intereses del centro y en un papel de oyente, sin realizar estudios $o$ investigaciones adicionales.

Algunas evidencias de formación compatible con el "Desarrollo profesional reflexivo". Profesorado que participa en ocasiones en actividades que coinciden con los objetivos del centro y realiza estudios e investigaciones con otros compañeros.

Formación ajustada al "Desarrollo profesional reflexivo". Profesorado que, de forma habitual, realiza actividades que emplean metodología activa, se junto a otros compañeros, que responden a objetivos de carácter comunitario (el centro o el profesorado de la especialidad) con estudios e investigaciones en equipo.

Una vez asignados los docentes en las categorías identificadas se realiza un análisis de frecuencias para conocer su distribución porcentual en función de su nivel de compatibilidad con el constructo "Desarrollo profesional reflexivo". 
El último objetivo del estudio consiste en identificar los factores o variables que se asocian al perfil de profesorado que mantiene un "Desarrollo profesional reflexivo". Para ello, el índice DPR es introducido como variable dependiente en un modelo jerárquicolineal (Raudenbush \& Bryk, 2002) para comprobar el ajuste de los datos empíricos al modelo teórico que se muestra en la Figura 1.

Figura 1. Modelo teórico de "Desarrollo profesional reflexivo"

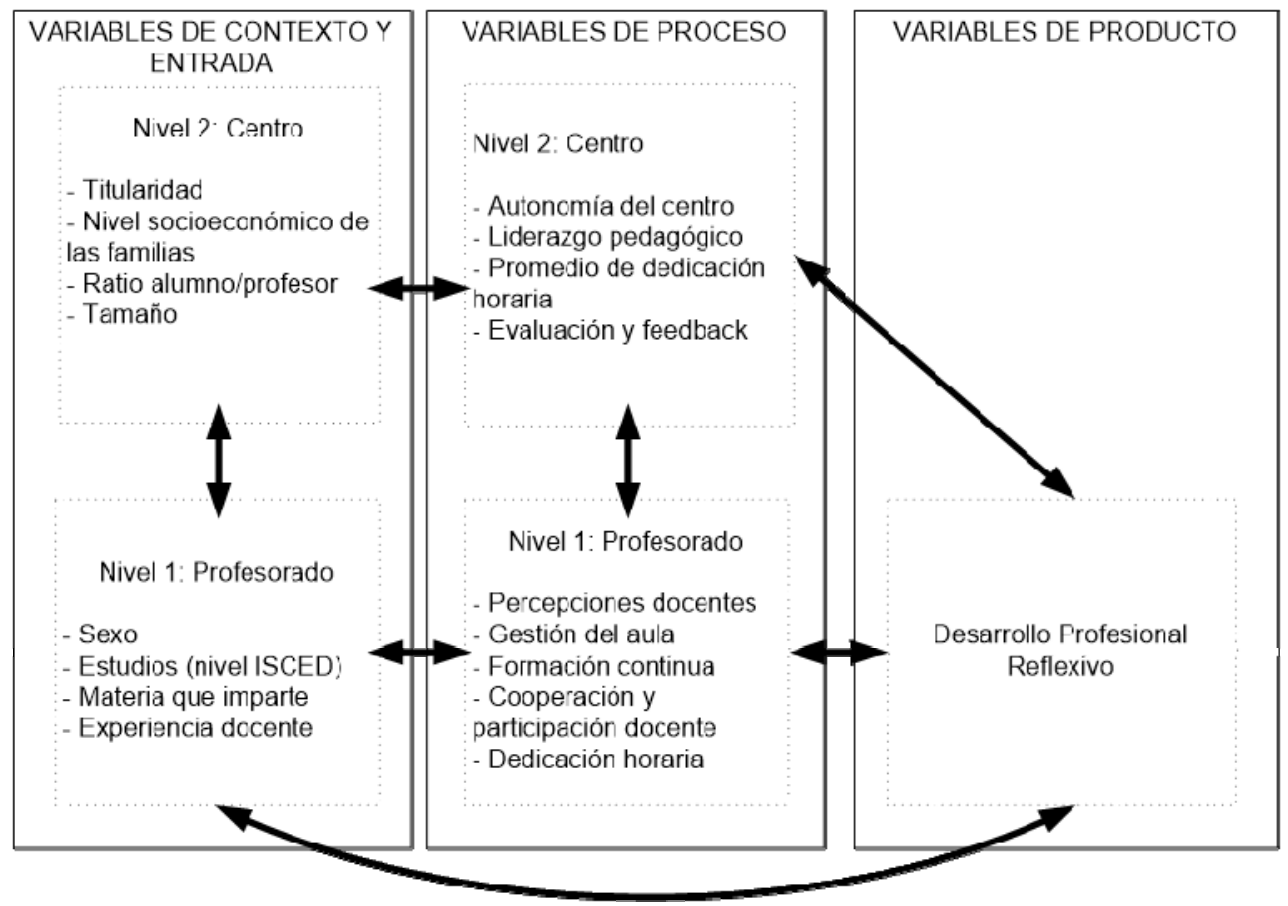

Este modelo se basa en los siguientes supuestos:

Existen docentes que participan sistemáticamente en actividades de desarrollo profesional reflexivo y tienen un plan de formación a medio y largo plazo, orientado al desarrollo de la organización educativa a la que pertenecen.

Mantienen ciertas características sociológicas (edad, experiencia, materia que imparte, etc.), psicológicas (percepciones de autocompetencia, creencias docentes, etc.) o profesionales (formación permanente, feedback, etc.).

Las características del centro educativo (tamaño, nivel educativo de las familias) y los procesos que ocurren en el mismo (autonomía, estilo de liderazgo, carga de trabajo, etc.) pueden facilitar o entorpecer el "Desarrollo profesional reflexivo".
Las características del docente y las del centro se relacionan e interaccionan, lo que obliga a emplear un modelo con dos niveles de medida de estas variables: el nivel individual (docente) y el nivel institucional (centro).

Existen muchos asertos que ilustran el último supuesto. Entre ellos, que el profesorado novel ocupa, por lo general, destinos periféricos y poco atractivos; que los centros pequeños tienen condiciones favorables para el desarrollo de proyectos integrales o que el estilo de dirección influye en la retroalimentación que recibe el profesorado.

\section{Resultados}

Los principales resultados del estudio están organizados de acuerdo con los objetivos planteados. En primer lugar se muestra la posición del profesorado español en relación al conjunto de países que participan en la encuesta TALIS. En segundo lugar, se 
presentan los análisis realizados para confirmar la estructura del índice de "Desarrollo profesional reflexivo". Posteriormente, se presentan los resultados del análisis multinivel que ajusta los datos disponibles al modelo teórico hipotetizado sobre el "Desarrollo profesional reflexivo" del profesorado. Finalmente, se identifican las características personales y de centro educativo asociadas a este perfil.

Situación del profesorado de España en las prácticas de "Desarrollo profesional efectivo"
El primer objetivo del estudio es analizar la situación de España en el contexto internacional, según el promedio de países que contempla el índice original estimado por TALIS. La Figura 2 presenta los países participantes ordenados según su promedio en el índice TEFFPROS. Según se aprecia, el promedio de España es significativamente inferior al promedio de TALIS. La puntuación es significativamente superior a dieciséis países, inferior a otros diez y no presenta diferencias significativas con seis de ellos. No aparece una pauta clara puesto que en los tres grupos figuran países con una gran diversidad social, cultural y económica.

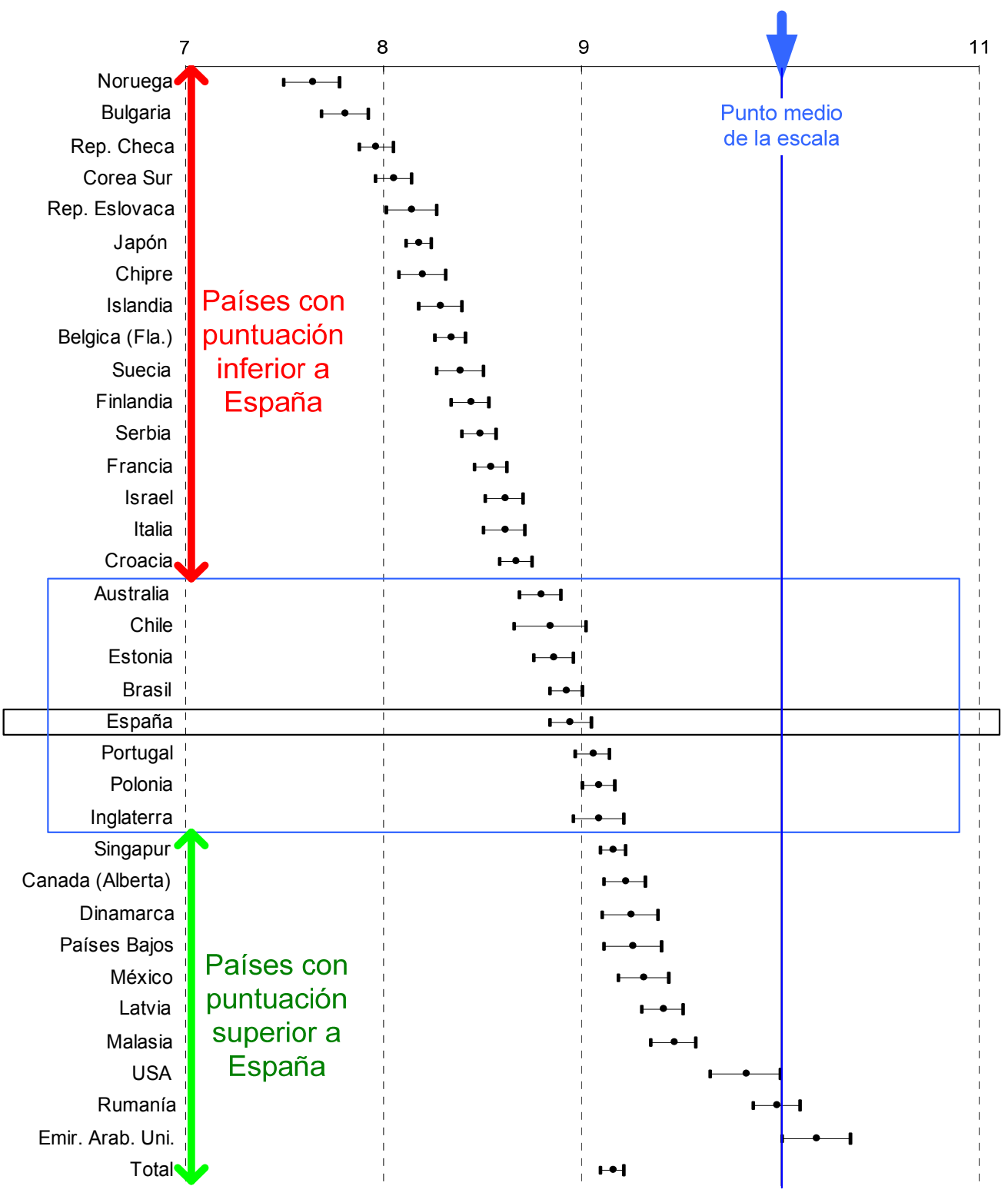

Figura 2. Situación de España en el índice internacional "Desarrollo profesional efectivo" (TEFFPROS) 
En la Figura 2 también se señala el punto medio de la escala Likert tras reescalar los resultados, es decir, la puntuación a partir de la cual el profesorado tiende a estar más de acuerdo con las afirmaciones que conforman este índice. En el conjunto de los países, las prácticas de desarrollo profesional para el empleo de métodos de aprendizaje activo requieren la realización de actividades de investigación con otros profesores y que estas se desarrollen durante un largo periodo de tiempo.

Para verificar las afirmaciones contenidas en el párrafo anterior, se seleccionaron aquellos docentes con una puntuación similar al promedio de TALIS. Con el profesorado seleccionado, equivalente a más de 50.000 docentes de los países participantes, se analizó la distribución de frecuencias de las cuatro cuestiones planteadas. El análisis muestra las características de las actividades de formación en las que participa el profesorado con puntuaciones en torno al promedio internacional. Estas son:

Participar en actividades de desarrollo profesional conjuntamente con compañeros del mismo centro o de la materia que imparte es una práctica que aún no está generalizada (sólo uno de cada cuatro docentes). En otras palabras, el profesorado participa en actividades de desarrollo profesional que no están conectadas con los intereses del centro.
La formación no suele basarse en métodos de aprendizaje activo ya que la mitad de los docentes indica que las actividades en las que participa suele adoptar un papel pasivo, limitándose a escuchar a un experto o conferenciante.

Las actividades de desarrollo profesional carecen o, en el mejor de los casos sólo tienen un pequeño componente de investigación (sólo uno de cada cuatro docentes).

La formación suele ser puntual o limitada en el tiempo. Tres de cada cuatro docentes indican que nunca (o sólo a veces) las actividades de formación se desarrollan a lo largo del tiempo.

\section{Construcción del índice "Desarrollo profesional reflexivo"}

El estudio exploratorio inicial ofrece garantías de que es posible reducir las cuatro cuestiones planteadas en el cuestionario TALIS a un índice sintético. Se muestran los pesos de cada ítem en el índice de "Desarrollo profesional reflexivo" y las correlaciones entre los ítems. El análisis confirmatorio se realiza mediante el ajuste de un modelo de ecuaciones estructurales, y tanto el análisis exploratorio como el confirmatorio permiten resumir los datos en una única dimensión abriendo así la vía para ajustar los datos a un modelo de respuesta al ítem.

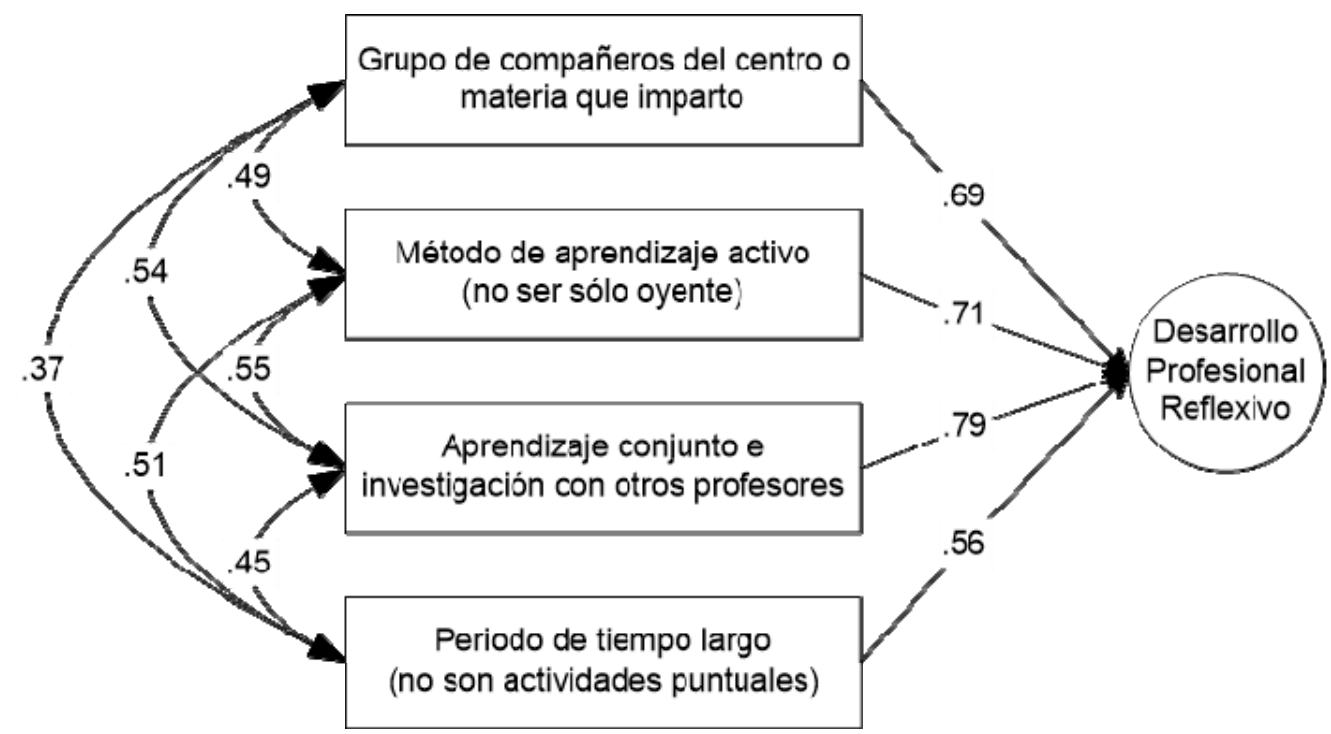

Figura 3. Resumen del ajuste de los ítems para la construcción del índice DPR 
Incidencia del perfil de "Desarrollo profesional reflexivo"

A continuación se muestra la Curva Característica del Test del índice DPR. Sobre ella se superpone la distribución de frecuencias según la puntuación alcanzada por el profesorado. Se han establecido dos puntos de corte, definidos a priori en función de las posibles respuestas a los cuatro ítems de la escala, y que generan los grupos siguientes: profesorado en cuyo estilo de enseñanza apenas hay rastro de actividades compatibles con el "Desarrollo profesional reflexivo", profesorado que presenta algunos indicios de dichas prácticas, y profesorado en el que predomina una mayor inclinación por la práctica docente reflexiva.

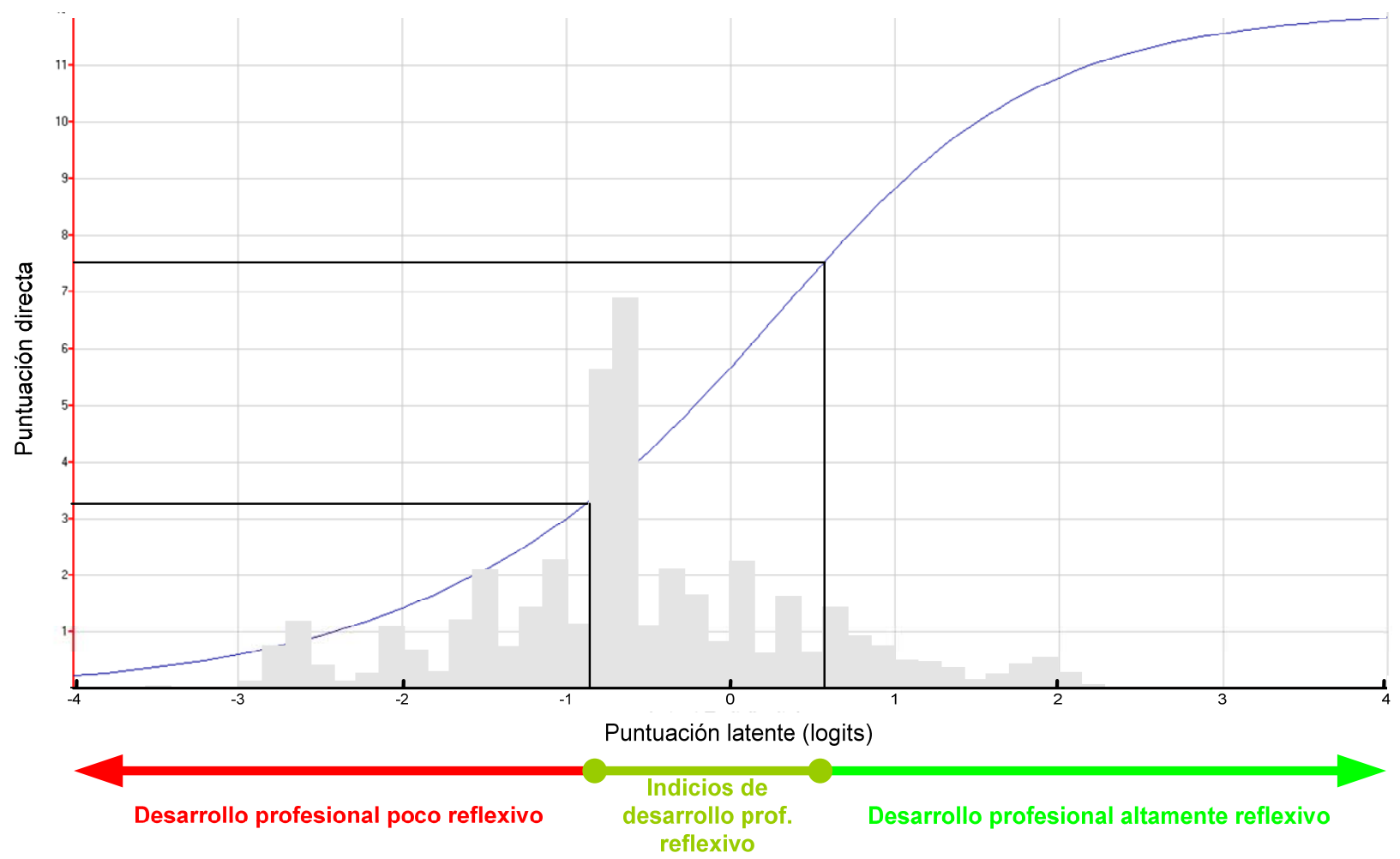

Figura 4. Curva Característica del Test "Desarrollo profesional reflexivo"

Establecidos los puntos de corte, se ha obtenido la distribución porcentual de las categorías definidas a partir de la escala. Se observa que tres de cada diez profesores o profesoras no presentan un "Desarrollo profesional reflexivo”. La situación más habitual (aproximadamente la mitad de la muestra), es la del profesorado cuyas actividades de desarrollo profesional se pueden considerar, en ocasiones, reflexivas, mientras que el grupo de docentes cuyo desarrollo profesional es claramente reflexivo es minoritario.

Así pues, en la situación considerada óptima hay pocos profesores. Aunque la mitad del profesorado se sitúa en una posición intermedia aceptable, resulta destacable que casi un tercio presente un desarrollo profesional tan alejado del planteamiento reflexivo.

\section{Factores asociados al perfil de "Desarrollo profesional reflexivo"}

En este punto se exponen los resultados del análisis jerárquico-lineal realizado para identificar los factores contextuales, de entrada y de proceso, correspondientes a los niveles personal e institucional relacionados con el perfil DPR.

La estrategia planteada considera cuatro modelos. Un modelo nulo, sin variables independientes, y tres modelos en los que se 
van introduciendo variables de contexto y entrada, variables de proceso de nivel individual y variables de proceso de nivel institucional.

El modelo nulo indica que el perfil reflexivo es un atributo individual del profesorado puesto que la gran mayoría de la varianza total del índice "Desarrollo profesional reflexivo" (en concreto el 94,8\%) se debe a diferencias entre el profesorado, mientras que únicamente el $5,2 \%$ se imputa a diferencias entre los centros.

En el modelo uno se incluyen las variables de entrada y contexto (Figura 1), es decir, los factores antecedentes. Este modelo explica poca varianza; algo más del $4 \%$ de las variaciones entre los centros, y menos del $2 \%$ de la varianza entre el profesorado. En consecuencia, las características sociológicas del profesorado y el resto de variables antecedentes mantienen cierta independencia con el DPR a nivel institucional (centro) y prácticamente nula con el desarrollo de este perfil a nivel individual (profesorado). En todo caso, los resultados indican que el DPR es más probable entre las mujeres, el profesorado con más experiencia y con titulación máxima de licenciatura (frente a los doctores u otras titulaciones por debajo del nivel CINE 5). En cuanto a los factores de contexto de centro el DPR tiende a ser mayor en aquellos centros de ratio más pequeña, lo que sugiere que una excesiva carga docente puede entorpecer la reflexión sobre la práctica.

El modelo dos incorpora las variables de proceso incluidas en el nivel de profesorado, además de las variables del modelo anterior. Muestra una capacidad predictiva mayor que aquel y resulta bastante más revelador en cuanto a resultados. Las variables contenidas en el mismo explican aproximadamente la mitad de las diferencias entre los centros y algo más del $11 \%$ de las diferencias entre el profesorado. Las características profesionales que reflejan las variables del modelo dos parecen ser el conjunto más determinante para explicar el DPR. Los resultados corroboran la relativa independencia entre el "Desarrollo profesional reflexivo” y las características sociológicas del profesorado y de los centros.

El modelo tres incluye los procesos medidos a nivel de centro. No aporta ganancia explicativa en la varianza entre centros y sí un $10 \%$ a la varianza entre profesorado. Las dos variables que resultan significativas, el liderazgo pedagógico y el uso de la evaluación para dar retroalimentación, apuntan a que aquellos centros con un liderazgo educativo fuerte y que usan los datos de las evaluaciones docentes para dar retroalimentación sobre las prácticas educativas, facilitan el desarrollo de estos procesos de reflexión docente.

De acuerdo con estos resultados, el "Desarrollo profesional reflexivo" se considera vinculado fundamentalmente a la formación continua del profesorado. Así, la variable con mayor efecto sobre la variable dependiente es la participación en una red de desarrollo profesional. El índice también aparece positivamente vinculado a aquel profesorado que tiene prioridades claras en cuanto a sus necesidades de formación y se relaciona negativamente con aquellos que señalan más dificultades para participar en actividades de formación continua. En su conjunto, la introducción en el modelo dos de estas tres variables muestra que el profesorado que participa con mayor frecuencia en actividades de formación permanente tiene más oportunidades de incorporar la reflexión a su proceso docente.

Por otro lado, el modelo dos indica, también, que el profesorado que puntúa más alto en la escala DPR participa con más frecuencia en actividades de cooperación docente y percibe mayores oportunidades en la implicación en la vida del centro. Esto pone de manifiesto que la colaboración docente y la corresponsabilidad en la toma de decisiones son prácticas vinculadas al "Desarrollo de pensamiento reflexivo”, aunque también aparece vinculado a una mayor carga de trabajo total, lo que parece coherente con el hecho de que la reflexión sobre la docencia implica un esfuerzo y una inversión en término de horas semanales de trabajo dedicadas a la profesión. 
También se constata cierta relación positiva entre el DPR y la eficacia escolar. Así, los docentes que puntúan más alto tienden a invertir menos tiempo de aula en actividades de control del orden y dedican más a las tareas de enseñanza y aprendizaje. Además, se autoperciben más eficaces a la hora de favorecer el aprendizaje de su alumnado.

Por último, la relación entre el "Desarrollo profesional reflexivo" y ciertas percepciones profesionales no ofrece resultados concluyentes. La única variable que vincula las percepciones docentes con el "Desarrollo profesional reflexivo" es la valoración positiva de las relaciones personales en el centro.

La lectura conjunta de los tres modelos permite concretar los siguientes resultados en relación al índice de "Desarrollo profesional reflexivo":

No es esperable encontrar un perfil nítido de centro asociado a un mayor "Desarrollo profesional reflexivo" del profesorado sino al quehacer individual del profesorado.

Los rasgos asociados con el DPR son la participación en redes de formación permanente, la cooperación docente $y$, en menor medida, las variables vinculadas a la eficacia instructiva, como las horas semanales de trabajo y ciertas percepciones docentes.

Aunque es difícil establecer un perfil de centro, existen ciertos indicios que relacionan la puntuación en el índice DPR con algunas características organizativas de los centros, como el papel del liderazgo y el uso de la evaluación para la mejora organizativa.

El conjunto de variables consideradas en este análisis explica poco más del $10 \%$ de la variación entre el profesorado y casi el $60 \%$ de las variaciones entre centros. Vemos, pues, que el "Desarrollo profesional reflexivo" es un constructo muy complejo cuya explicación requiere más análisis.

\section{Discusión y conclusiones}

El índice de "Desarrollo profesional reflexivo" resulta adecuado para el establecimiento de diferentes perfiles de profesorado cuyas características inciden en la efectividad de los procesos y a partir de los cuales se pueden desarrollar propuestas para los modelos de formación de los docentes.

De acuerdo con los objetivos que han orientado este estudio, las principales conclusiones se pueden resumir en las siguientes:

En el conjunto de los países participantes en TALIS, las respuestas del profesorado apuntan a que las actividades de formación responden a intereses particulares, tienen una duración corta y con papel pasivo de los participantes, sin plantearse el realizar actividades de estudio e investigación conjunta con otros profesores. España presenta un resultado significativamente más bajo que el promedio de países, aunque hay varios países próximos con resultados aún más bajos (p.ej. Italia, Francia, Suecia, Bélgica, etc.).

Los datos indican que el "Desarrollo profesional reflexivo" es minoritario. Aunque un porcentaje importante de docentes participa en actividades de formación con algunos rasgos propios del desarrollo reflexivo, las posibilidades de mejora son evidentes ya que aquellos que no tienen ningún ajuste al perfil representan casi a un tercio.

El profesorado con un perfil individual más propicio para el "Desarrollo profesional reflexivo" se caracteriza por su experiencia y estima docente, ser mujer y tener claras prioridades de formación e interés por la formación permanente. Su modelo de enseñanza es efectivo y maneja con eficacia y estilo activo los tiempos de aprendizaje de su alumnado.

Para incrementar la proporción de docentes cuyo perfil responsa a las características del profesional reflexivo (DPR), consideramos que su formación debe adaptarse a un modelo investigador con un enfoque activo, organizado en red y que trabaje sobre proyectos formativos de centro o muy vinculados a la materia docente. El modelo ideal precisa de la colaboración y cooperación de los docentes en un proyecto de centro al que dediquen su 
tiempo profesional y en el que participen de forma corresponsable en las decisiones organizativas. Tal como expresa Linda Darling-Hammond, profesora de la Standford University, en el prólogo del informe del Instituto Nacional de Educación de Singapur (NIE, 2009), los programas universitarios de formación del profesorado deben tener los siguientes atributos: una compactación de cursos que ofrezcan una experiencia formativa coherente; estándares bien definidos sobre prácticas y logros académicos; una programación básica centrada en el aprendizaje del alumnado, en la evaluación y el contenido pedagógico; metodología de enseñanza basada en problemas; evaluación activa mediante estudios de caso y portfolios; y prácticas con profesorado experto y ofertadas en los primeros cursos de la carrera.

Este modelo teórico de formación de profesorado reflexivo e investigador se puede ver aplicado, en algunos sistemas universitarios. Así, Finlandia pide a su profesorado una cualificación de Master; en Singapur se pretende mejorar la profesionalización de la enseñanza ofertando una línea de acceso que permita atraer a los estudiantes más brillantes hacia la profesión docente (el 10\% de mejor calificación accede a un itinerario de Graduado en Magisterio y Master).

El modelo de Singapur, se basa en tres principios básicos: conciencia de la diversidad de los estudiantes y que todos pueden aprender si tenemos en cuenta sus estilos y organizamos medios acordes con ellos; mantenimiento de altos objetivos de logro en relación con los rápidos cambios que se produce en la educación; y, en tercer lugar, el servicio a la profesión y a la comunidad, que requiere compromiso con el trabajo en colaboración y una actitud de mejora permanente en beneficio de la sociedad.

El programa de formación de profesorado de la University of Northern Iowa incluye un método de reflexión técnica (Etscheidt, Curran \& Sawyer, 2012). En una primera fase el alumnado planifica una unidad curricular que, una vez impartida, es sometida a una autoevaluación supervisada por un tutor en tres aspectos: planificación, desarrollo y defensa del resultado. La segunda fase del programa, centrada en la reflexión deliberativa (Lee, 2008), tiene por objetivo analizar situaciones alternativas y justificar decisiones mediante pensamiento divergente sobre creencias pedagógicas. Este objetivo se desarrolla mediante análisis de vídeos que permiten deconstruir acciones educativas del aula y la realización de un diario sobre sucesos concretos, por ejemplo evaluación del alumnado, relación entre el profesorado, control de comportamientos o relación familiacentro. Estos análisis son remitidos semanalmente al tutor, quien proporciona retroalimentación considerando posibles alternativas. En una tercera y última fase se incide en el análisis crítico de la situación socioeducativa concreta.

En estos modelos universitarios considerados de "buena práctica", el aprendizaje experiencial sitúa al futuro docente en el fenómeno a estudiar mediante actividades integradas en el Practicum. Para reforzar este aprendizaje experiencial y facilitar evidencias para el seguimiento y la evaluación se utiliza el e-portfolio y el proyecto profesional. El primero, permite que los estudiantes registren y elaboren reflexiones sobre su aprendizaje; el segundo es un requerimiento de graduación que responde a planteamientos prácticos que permiten evidenciar las competencias de analizar, transformar, crear y aplicar conocimientos; desarrollar de forma sistemática estudios sobre temas de enseñanzaaprendizaje, así como liderar la puesta en marcha y el desarrollo de comunidades de aprendizaje. Uno de los principales componentes de este proyecto es el portafolio profesional, que incluye una reflexión crítica sobre todo lo aprendido y trabajos individuales de diferentes modalidades.

Este modelo de formación del profesorado basado en el "Desarrollo profesional reflexivo" no está generalizado aunque los resultados 
obtenidos permiten intuir la importancia que en el futuro puede tener para la política educativa.

\section{Referencias}

Anderson, R. D. (2002). Reforming Science Teaching: what research says about inquiry. Journal of Science Teacher Education, 13(1), $1-12$ doi: http://dx.doi.org/10.1023/A:1015171124982

Cochran-Smith, M. \& Lyttle, S. L. (2009). Inquiry as Stance: Practitioner Research in the Next Generation. New York: Teachers College Press.

Dana, N. F. \& Yendol-Hoppey, D. (2009). The Reflective Educator's Guide to Classroom Research: Learning to Teach and Teaching to Learn Through Practitioner Inquiry. Thousand Oaks, CA: Corwin.

Darling-Hammond, L. \& Bransford, J. (Eds.) (2005). Preparing Teachers for a Changing World: What teachers should learnand be able to do. San Francisco, CA: Jossey-Bass.

Dewey, J. (1985 [1916]). Democracy and Education. Vol. 9 of John Dewey: The Middle Works, 1899-1924, edited by Jo Ann Boydston. Carbondale and Edwardsville, IL: Southern Illinois University Press

Etscheidt, S., Curran, Ch. M. \& Sawyer, C. M. (2012). Promoting Reflection in Teacher Preparation Programs: a multilevel model. Teacher Education and Special Education, 35(1), 7-26. doi: http://doi.org/10.1177/0888406411420887

Freese, A. R. (2006). Reframing one's teaching: Discovering our teacher selves through reflection and inquiry. Teaching and Teacher Education, 22, 100-119. doi: http://doi.org/10.1016/j.tate.2005.07.003

Hattie, J. (2003). Teachers Make a Difference: What is the Research Evidence? Comunicación presentada en el Australian Council for Educational Research Annual Conference on Building Teacher Quality, Melbourne. Consultado en: http://www.education.auckland.ac.nz/webdav/s ite/education/shared/hattie/docs/teachers-makea-difference-ACER-\%282003\%29.pdf
Jay, J. \& Johnson, K. (2002). Capturing complexity: A typology of reflective practice for teacher education. Teaching and Teacher Education, 18, 73-85. doi: http://doi.org10.1016/S0742-051X(01)00051-8

Kember, D., McKayb, J., Sinclairc, K. \& KamYuet Wong, F. (2008). A four-category scheme for coding and assessing the level of reflection in written work. Assessment \& Evaluation in Higher Education. 22(4), 369-37. doi: http://doi.org/10.1080/02602930701293355

Lee, I. (2008). Fostering preservice reflection through response journal. Teacher Education Quarterly, 35(1), 117-139. Consultado en http://www.jstor.org/stable/23479034

NCATE (2008). Professional standards for the accreditation of teacher preparation institutions. Washington, DC: National Council for Accreditation of Teacher Education. Consultado en http://www.ncate.org/documents/standards/NC ATE\%20Standards\%202008.pdf

Newel, S. T. (1996). Practical inquiry: collaboration and reflection in teacher education reform, Teaching and Teacher Education, 12(6), 567-576. doi: https://doi.org/10.1016/S0742051X(96)00001-7

Nie (2009). A Teacher Education Model for the $21^{\text {st }}$ Century. Singapore: National Institute of Education.

OECD (2014). TALIS Results: An international perspective on teaching and learning. TALIS, OECD Publishing. Doi: http://doi.org/10.1787/97892641962261-en

Raudenbush, S. W. \& Bryk, A. S. (2002). Hierarchical Linear Models: Applications and Data Analysis Methods. $2^{\text {nd }}$ edition. Newbury Park, CA: Sage.

Schön, D. A. (1987). Educating the Reflective Practitioner. San Francisco, CA: Jossey-Bass.

Rust, F. O. (2007). Action research in early childhood contexts. En J. A. Hatch (ed.), Early Childhood Qualitative Research, 95-108. New York. Routledge

Scruggs, T. E. \& Mastropieri, M. A. (1994). The construction of scientific knowledge by 
students with mid disabilities. Journal of Special Education, 28, 307-321. Doi: https://doi.org/10.1177/002246699402800306

Sparks-Langer, G. M. \& Berstein, A. (1991). Synthesis of Research on Teachers' Reflective Thinking. Educational Leadership, 48(6), 3744.

Stremmel, A. J. (2007). The Value of Teacher Research. Nurturing Professional and Personal
Growth through Inquiry. Voices of practitioners, 2(3), 1-9

Ward, J. \& McCotter, S. (2004). Reflection as a visible outcome for preservice teachers. Teaching \& Teacher Education, 20(3), 243257. doi: http://doi.org/10.1016/j.tate.2004.02.004

\section{Nota}

[1] Las referencias pedagógicas sobre la formación del profesorado como investigador, indagador y profesional reflexivo son abundantes en revistas científicas de Educación como The Journal of Inquiry \& Action Education (http://digitalcommons.buffalostate.edu/jiae/vol4/iss3/), Studying Teacher Education: a journal of self-study of teacher education practice (http://www.tandfonline.com/loi/cste20\#.Uo3ibyduGKw), o Networks: an on-line journal for teacher research (http://journals.library.wisc.edu/index.php/networks).

\section{$\underline{\text { Agradecimientos }}$}

Este trabajo ha sido posible gracias al apoyo recibido por parte del Instituto Nacional de Evaluación Educativa del Ministerio de Educación, Cultura y Deporte, y de la Consejería de Educación y Cultura del Gobierno del Principado de Asturias. 
Fernández-Fernández, Samuel; Arias-Blanco, José-Miguel; Fernández-Alonso, Rubén; Burguera-Condon, Joaquín \& Fernández-Raigoso, Marcelino (2016). Pensamiento reflexivo e investigador en educación. Aspectos a tener en cuenta en la formación del profesorado. RELIEVE, 22(2), art. 3. doi: http://dx.doi.org/10.7203/relieve.22.2.8425

\section{Authors / Autores}

To know more /

Saber más

Fernández-Fernández, Samuel (samuel@uniovi.es).

Catedrático de Universidad de la Universidad de Oviedo, Dpto. de CC de la Educación. Área de Métodos de Investigación y Diagnóstico en Educación (MIDE). Mantiene una línea de investigación sobre Calidad y Evaluación Educativa. En los últimos años ha dirigido un proyecto europeo (QAHECA) y otro regional sobre absentismo escolar. Evaluador nacional e internacional de programas universitarios de Educación, ha publicado artículos sobre desarrollo metodológico en evaluación de programas en atención a personas con discapacidad. Dirección postal: Facultad de Formación del Profesorado y Educación. C/Aniceto Sela s/n, 33005-Oviedo, Asturias (España)

Arias-Blanco, José-Miguel (arias@uniovi.es)

Doctor en Pedagogía por la Universidad de Oviedo. Profesor Titular de Universidad del área MIDE de la Universidad de Oviedo. Sus principales líneas de investigación son: metodología de la investigación en ciencias sociales, competencias profesionales, metodología de enseñanza y aprendizaje e innovación en la educación superior, evaluación de la educación superior. Dirección postal: Facultad de Formación del Profesorado y Educación. C/Aniceto Sela s/n, 33005, Oviedo, Asturias (España).

Fernández-Alonso, Rubén fernadezaruben@uniovi.es.

Doctor en Psicometría por la Universidad de Oviedo. Coordinador de Estudios de Evaluación y Calidad en la Consejería de Educación y Cultura del Gobierno del Principado de Asturias y profesor asociado del Departamento de Ciencias de la Educación de la Universidad de Oviedo. Sus principales líneas de investigación y especialización son: los modelos de análisis aplicados a la evaluación del sistema educativo, la construcción de índices e indicadores educativos y los deberes escolares. Dirección postal: Consejería de Educación y Cultura del Gobierno del Principado de Asturias. Plaza de España, 5, 4, 33005 - Oviedo, Asturias (España).

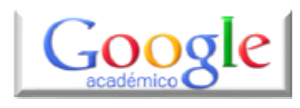

Burguera-Condon, Joaquín (burguera@uniovi.es).

Doctor en Pedagogía por la Universidad de Oviedo. Profesor Ayudante Doctor de Universidad, del Departamento de Ciencias de la Educación, en el Área MIDE en la Universidad de Oviedo. Sus principales líneas de investigación son: prácticas externas-practicum, metodología e innovación docente en educación, metodología de investigación en ciencias sociales, evaluación de programas socioeducativos y tutoría y orientación educativa. Dirección postal: Facultad de Formación del Profesorado y Educación. C/Aniceto Sela s/n, 33005 - Oviedo, Asturias (España).

\section{Fernández-Raigoso, Marcelinoraigoso@unovi.es}

Titular de Escuela Universitaria por la Universidad de Oviedo en el área MIDE del Departamento de Ciencias de la Educación. Coordinador de investigaciones sobre necesidades formativas y competencias en diferentes ámbitos profesionales. Miembro de grupos de investigación sobre competencia digital, las prácticas en la educación superior y la mejora de las actividades profesionales en educación. Ha realizado estudios relativos a la inserción profesional de los universitarios, tanto a nivel autonómico como europeo. Dirección postal: Facultad de Formación del Profesorado y Educación. Despacho 312. C/Aniceto Sela s/n, 33005- Oviedo,

ORCID 0000-0002-4963-2362

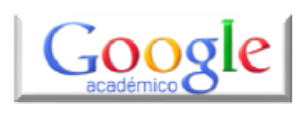

ResearchGate Asturias (España). 
Fernández-Fernández, Samuel; Arias-Blanco, José-Miguel; Fernández-Alonso, Rubén; Burguera-Condon, Joaquín \& Fernández-Raigoso, Marcelino (2016). Pensamiento reflexivo e investigador en educación. Aspectos a tener en cuenta en la formación del profesorado. RELIEVE, 22(2), art. 3. doi: http://dx.doi.org/10.7203/relieve.22.2.8425

\section{RELIEVE}

\section{Revista ELectrónica de Investigación y EValuación Educativa E-Journal of Educational Research, Assessment and Evaluation}

[ISSN: 1134-4032]

(C) Copyright, RELIEVE. Reproduction and distribution of this articles it is authorized if the content is no modified and their origin is indicated (RELIEVE Journal, volume, number and electronic address of the document).

(C) Copyright, RELIEVE. Se autoriza la reproducción y distribución de este artículo siempre que no se modifique el contenido y se indique su origen (RELIEVE, volumen, número y dirección electrónica del documento). 